\title{
Drug Dosing During Continuous Renal Replacement Therapy
}

\author{
Mariann D. Churchwell* and Bruce A. Mueller† \\ *Department of Pharmacy Practice, University of Toledo, College of Pharmacy, Toledo, Ohio, and \\ tDepartment of Clinical, Social and Administrative Sciences, University of Michigan College of Pharmacy, \\ Ann Arbor, Michigan
}

\begin{abstract}
Continuous renal replacement therapy (CRRT) has given clinicians an important option in the care of critically ill patients. The slow and continuous dialysate and ultrafiltrate flow rates that are employed with CRRT can yield drug clearances similar to an analogous glomerular filtration rate of the native kidneys. Advantages such as superior volume control, excellent metabolic control, and hemodynamic tolerance by critically ill patients are well documented, but an understanding of drug dosing for CRRT is still a bit of a mystery. Although some pharmaceutical companies have

dedicated postmarket research in this direction, many pharmaceutical companies have chosen not to pursue this information as it is not mandated and represents a relatively small part of their market. This lack of valuable information has created many challenges in the care of the critically ill patient as intermittent hemodialysis drug dosing recommendations cannot be extrapolated to CRRT. This drug dosing review will highlight factors that clinicians should consider when determining a pharmacotherapy regimen for a patient receiving CRRT.
\end{abstract}

Unlike intermittent hemodialysis (IHD), continuous renal replacement therapy (CRRT) provides relatively constant and predictable drug clearance. The dialysate and/or ultrafiltrate flow rates that are employed can yield drug clearance similar to an analogous glomerular filtration rate (GFR) of the native kidneys. Obviously, the CRRT system does not account for the secretion and reabsorption of the native kidney, but standard firstorder drug clearance equations can be used to calculate dosing regimens. Drug clearance by pumped CRRT is slow, yet constant; consequently determining drug dosing regimens should be "easier" than IHD. However, developing a drug dosing regimen for a patient receiving CRRT is not always as easy as it seems.

Depending on the mode of CRRT, the combined dialysate and ultrafiltrate flow rates have been recommended as a rough estimate of creatinine clearance $(\mathrm{CrCl})(1)$. This method provides a starting point for initial drug dosing estimations. Although an estimate upon which to base empiric dosing, it may not be an appropriate basis for extended durations of drug therapy. Critically ill patients with acute kidney injury

Address correspondence to: Bruce A. Mueller, PharmD, FCCP, Professor and Chair, Department of Clinical, Social and Administrative Sciences, University of Michigan College of Pharmacy, 428 Church Street, Ann Arbor, Ml 48109-1065, or e-mail: muellerb@umich.edu.

Seminars in Dialysis-Vol 22, No 2 (March-April) 2009 pp. 185-188

DOI: $10.1111 /$ j. 1525-139X.2008.00541.x

(C) 2009 Wiley Periodicals, Inc.
(AKI) are dynamic. Renal function, and consequently drug clearance, may be continually changing. Patientspecific, nonrenal factors such as inflammation, hepatic and other organ system function, and changes in volume status all may change profoundly in an AKI patient receiving CRRT. Drug metabolism in patients with AKI can be quite different from what is observed in healthy patients with normal renal function $(2,3)$ or even from patients with chronic kidney disease (4). Further, renal replacement therapy itself can affect drug metabolism (5). All of these factors can have a considerable effect on drug disposition, and consequently, the appropriate drug dosing regimens.

Many CRRT-specific factors have obvious effects on drug removal. In many cases these aspects of CRRT are recognized by clinicians when determining drug dosing, and have been well described in the literature. For example, dialysate/ultrafiltrate flow rates have considerable influence on drug clearance for most drugs (6). The use of higher permeability hemodiafilters will result in considerably higher drug clearance rates than less permeable membranes, especially for drugs of middle molecular weight (MW) size like vancomycin (MW 1450 Da) (7) or daptomycin (MW 1620 Da) (8).

Less studied and less recognized by clinicians are some other CRRT technique-specific factors that influence drug clearance. For example, in CRRT systems with a convective component, placement of the replacement solutions has an important impact on solute clearance. Uchino et al. (9) reported that vancomycin sieving coefficient steadily decreased as the proportion of replacement solutions administered prefilter vs. postfilter, decreased. 
Prefilter replacement fluid dilutes the blood that is presented to the hemodialyzer and decreases the amount of drug that is cleared. Consequently, in the trial by Uchino where the ratio of prefilter and postfilter fluid replacement was varied, vancomycin clearance was lowest when all fluids were given prefilter and almost $25 \%$ higher when the majority of the fluids were given postfilter. The prefilter or postfilter placement of replacement solutions tends to be unit dependent. Where in the CRRT system the replacement solutions are given could account for many of the differences found in published drug sieving coefficients and clearance rates. Brunet et al. (10) suggested that there was a $15-19 \%$ clearance reduction for urea and creatinine when ultrafiltrate replacement solutions were administered prefilter.

Another CRRT system-related factor that is usually not considered much by clinicians when assessing CRRT drug clearance is hemodiafilter age. Early data suggest that hemodiafilter membrane performance changes over time $(11,12)$. Drug sieving coefficients are at their highest when the hemodiafilter is first put into use. The sieving coefficient declines with time, ostensibly because of a growing protein layer that builds up on the membrane surface and/or because of a decline in the number of unclotted hollow fibers $(11,12)$. In the earlier days of CRRT, most CRRT centers limited the amount of time that hemodiafilters could be used. Indeed, hemodiafilter package inserts typically stated that they should not be used beyond 48 hours. However, today it is not uncommon for some units to use CRRT circuits for as long as 72-96 hours, in spite of the hemodiafilter manufacturer warnings. Likely the clearance of very small solutes like urea and creatinine are not greatly affected by increasing the lifespan of a CRRT circuit, but clearance of drugs with larger MWs may be reduced as these hemodiafilters become "clogged" over time. This aspect of CRRT drug clearance has not been studied extensively, and most published pharmacokinetic studies and CRRT case reports never mention how long the hemodiafilter was used when the measurements were conducted.

It is interesting to note how "uniform" the practice of IHD has become compared with the practice of CRRT. While dialyzers and machines may differ between units, most dialysis centers and hospitals conduct the hemodialysis procedure itself similarly. They use the same types of vascular access, treatment durations, anticoagulation, and similar blood and dialysate flow rates. This uniformity has made drug dosing relatively predictable and manageable. Many references exist for clinicians to use to make informed decisions on pharmacotherapy. In contrast, the practice of CRRT has tremendous variability. Despite many valiant efforts like the Acute Dialysis Quality Initiative, little agreement has been reached as to the optimal method to deliver CRRT. Indeed, a question as fundamental as whether diffusive or convective modalities should be used is still fiercely debated.

Clinicians often make the assumption that CRRT drug clearance is pretty much the same whether continuous venovenous hemodialysis (CVVHD), continuous venovenous hemofiltration $(\mathrm{CVVH})$, or the combination, continuous venovenous hemodiafiltration (CVVHDF) is used. With conventional CRRT at low dialysate/ultrafiltrate production rates $(<1$ //hour) this assumption is likely true. However, multiple studies show that dialytic therapies will always have inferior solute clearance to convective therapies at the same dialysate/ultrafiltrate flow rates. The clearance differences become larger as the dialysate/ultrafiltrate flow rates increase and as the MW of the solute increases. This rule of thumb should guide your estimates of drug clearance:

The drug clearance at any given dialysate/ultrafiltrate flow rate is:

$$
\text { CVVH }>\text { CVVHDF > CVVHD }
$$

The clearance difference might be relatively small for solutes like urea, but for larger molecules like vancomycin or daptomycin, the difference is marked $(7,8)$.

\section{Patient Case}

An approach toward drug dosing is best conveyed by examining a patient case and discussing how one would determine various drug regimens. Take, for example, an elderly male who developed AKI following abdominal surgery. CVVHDF with prefilter replacement fluid was initiated to remove edema and to correct an electrolyte imbalance. The CRRT flow rates are set at a blood flow of $200 \mathrm{ml} /$ minute, dialysate flow of $21 /$ hour (33 $\mathrm{ml} / \mathrm{minute}$ ), and an ultrafiltrate production rate of $500 \mathrm{ml} /$ hour $(8 \mathrm{ml} /$ minute). His pertinent dosing information includes his weight $232 \mathrm{lbs}(105.5 \mathrm{~kg})$ with an estimated preadmission "dry" weight of $188 \mathrm{lbs}$ $(85.5 \mathrm{~kg})$ and the fact that the patient is oliguric $(200 \mathrm{ml}$ urine/ 24 hours). An empiric estimate of creatinine clearance attributed to CVVHDF is $\sim 41 \mathrm{ml} /$ minute (dialysate flow rate + ultrafiltrate production rate). This estimate is likely a slight overestimation by 6-8 $\mathrm{ml} /$ minute because the replacement solution is being given prefilter. Obviously, if CRRT were stopped for any period of time, this patient's estimated clearance would be $<10 \mathrm{ml} /$ minute, as evidenced by his low urine output. At the initiation of CVVHDF, the patient has spiked a fever. The medical team is suspecting a bacteremia and wants to initiate a broad spectrum empiric therapy regimen of gentamicin, daptomycin, and "Drug X."

Understanding potential drug removal characteristics by these continuous therapies can simplify one's approach to drug dosing. Knowledge of each drug's pharmacokinetic and pharmacodynamic properties enables the clinician to empirically drug dose but also adjust the drug's dose as the patient's status changes. The clinician must also establish a goal for each therapy and monitor for therapeutic success, failure, and adverse effects.

\section{Gentamicin}

Gentamicin has a MW $\sim 477 \mathrm{Da}$ and a volume of distribution $\left(V_{\mathrm{d}}\right)$ of $\sim 0.25$ in healthy adults and protein binding rate is only $\sim 10-20 \%$ (13). Because of these characteristics, it is easily removed from the blood by CRRT without much regard to hemodialyzer membrane type or size. The so-called "once daily 
aminoglycoside regimens" $(14,15)$ take advantage of gentamicin's concentration-dependent antibacterial activity and post-antibiotic effect, but these regimens are reserved for patients with estimated GFR $>50 \mathrm{ml}$ minute. Consequently, in this patient, traditional gentamicin dosing methods would be used to achieve peak serum concentrations of 6-8 mg/1. This peak concentration should be sufficient to exceed the minimum inhibitory concentration $\left(\mathrm{MIC}_{90}\right)$ of the organism.

Gentamicin has serious adverse effects associated with drug accumulation, so it is imperative that an ideal dosing regimen includes a period of time where the drug concentration is below 1-2 $\mu \mathrm{g} / \mathrm{l}$. Based on this patient's body weight, $V_{\mathrm{d}}$ and estimated drug peak concentration, a loading dose of $2-2.5 \mathrm{mg} / \mathrm{kg}$ followed by $1-1.5 \mathrm{mg} / \mathrm{kg}$ every 24 hours would be an appropriate initial dose. As edema is removed and the patient approaches his dry weight the gentamicin dose must be adjusted. Whenever possible, serum drug concentrations should be obtained once the drug has had time to distribute. Generally, a peak concentration should be obtained 30 minutes after the end of the infusion and a trough concentration before the next scheduled dose begins. Usually, serum concentrations would not be necessary after the first dose of gentamicin but this patient has a variable $V_{\mathrm{d}}$ with unstable renal function and checking serum concentrations after the first dose would be warranted in this case. Because this patient will likely have a reduction in volume of distribution as euvolemia is attained, gentamicin peak and trough serum concentrations should be obtained no less than once weekly.

\section{Daptomycin}

Daptomycin has a $V_{\mathrm{d}} 0.11 / \mathrm{kg}$ which is smaller than gentamicin but its MW of $1650 \mathrm{Da}$ is considerably larger. It is highly protein bound $(90-92 \%)$ in healthy adults (16). Daptomycin removal by CVVHDF poses an interesting problem as most of the drug remains in the vasculature (as evidenced by its small $V_{\mathrm{d}}$ ), which increases the probability of its CVVHDF removal despite its high protein binding rate. Daptomycin's large MW may slow the drugs movement across a hemodialyzer membrane but the nonprotein bound daptomycin is still small enough that it will readily cross highly permeable CRRT membranes (8). The manufacturer's daptomycin dosing recommendations for bacteremia in patients with a creatinine clearance $>30 \mathrm{ml} /$ minute is $6 \mathrm{mg} / \mathrm{kg}$ every 24 hours and every 48 hours with a creatinine clearance $<30 \mathrm{ml} /$ minute (17). CVVHDF is providing an estimated creatinine clearance of $\sim 35 \mathrm{ml} /$ minute for this patient and the clinician may be tempted to select a dose of $6 \mathrm{mg} / \mathrm{kg}$ every 24 hours. Based on data from our in vitro continuous dialysis study and early results of an ongoing clinical trial, we would suggest a dose of $8-10 \mathrm{mg} / \mathrm{kg}$ every 48 hours $(8,18)$.

\section{Drug X}

Drug X represents a drug that has recently been brought to market and for which there are no published studies of this drug's disposition in CRRT. The "literature" indicates that unlike gentamicin and daptomycin, which have a substantial post-antibiotic effect $(19,20)$, Drug X's pharmacodynamic properties require that the drug concentration must be continuously maintained above the $\mathrm{MIC}_{90}$ for the drug to be effective and decrease the chance of a resistant organism forming. Other drugs with this pharmacodynamic profile include $\beta$-lactam antibiotics, for example.

What information regarding Drug $\mathrm{X}$ do you need to identify to dose this drug? Each drug's distinctive pharmacokinetic and pharmacodynamic property must be considered when dosing to obtain the best possible outcomes. An understanding of the pharmacodynamic goals of the drug and then applying the drug's pharmacokinetics will assist in developing a dosing strategy for drugs with limited available data. Often, all of the pertinent pharmacokinetic data can be found in the manufacturer's package insert. Drug characteristics such as $V_{\mathrm{d}}$, MW, and protein binding rate are important considerations in determining how much of Drug $\mathrm{X}$ will be removed by CRRT. A large $V_{\mathrm{d}}(>11 / \mathrm{kg})$ decreases the likelihood that a drug will be substantially removed by hemodialysis or CRRT assuming enough time for the drug to distribute. High-flux hemodialyzers allow for the removal of drugs with a MW of $\leq 2000 \mathrm{Da}$. Finally, a drug that is highly protein bound is less likely to be removed than one that is mostly unbound as only the nonprotein bound drug can cross the hemodiafilter membrane. The unbound fraction of the drug (1-protein binding rate $=$ unbound fraction) multiplied by the dialysate/ultrafiltrate rate yields the drug's clearance rate.

With this information, an estimate of the likely removal of Drug X by CRRT can be made. If the clearance of the drug by CRRT is $<25 \%$ of the total body clearance, dosing adjustment for CRRT is probably unnecessary. If CRRT looks to be an important source of drug clearance, a typical initial loading dose would be given, and maintenance doses would likely be similar to that given to a patient with a creatinine clearance of 30 $50 \mathrm{ml} /$ minute. If serum drug concentrations can be obtained in a timely manner to guide therapy, it should be done. As the patient's volume status changes, doses should similarly be adjusted.

There are several reliable drug dosing resources that should be consulted including the Renal Drug Dosing Handbook (21) and software packages such as Micromedex (22). Despite these resources, realize that few drugs have been studied in this regard. One of us (Mueller) were involved in the preparation of the CRRT drug dosing recommendations in the Drug Prescribing in Renal Failure book. Our purpose was to make evidencebased CRRT dosing recommendations and grade the evidence upon which we made decisions. Interestingly, of the $\sim 475$ drugs that were evaluated for entry into the book, less than $20 \%$ of those drugs had any type of published CRRT pharmacokinetic studies conducted on them. Consequently, most of the recommendations had to be made using knowledge of pharmacokinetic and extracorporeal solute removal principles as outlined above. 
Even in published drug dosing recommendations for patients not receiving CRRT, dosing recommendations differ between textbooks. Vidal et al. (23) reviewed the renal drug dosing recommendations of four commonly used published dosing references. The authors found a surprising amount of disagreement between the four reputable references for 100 commonly prescribed drugs: British National Formulary (2004), Martindale: The Complete Drug Reference (2004), AHFS Drug Information (2004), Drug Prescribing in Renal Failure (1999). Each drug dosing resource used a different description of renal impairment with little agreement among categories. The authors also found several instances where one resource stated a drug required no dosage adjustment but another resource indicated the same drug as contraindicated in renal impairment. More than one dosing resource should be used when available as dosing evidence remains sparse in this area. In a follow-up letter to this paper, Aronoff (24) described drug dosing in patients with renal disease as well as we have heard it summarized:

"Despite numerous secondary sources of drug dosing information, drug prescribing in renal failure remains imprecise and relies on interpolation, extrapolation, and estimation."

\section{References}

1. Joy MS, Matzke GR, Armstrong DK, Marx MA, Zarowitz BJ: A primer on continuous renal replacement therapy for critically ill patients. Ann Pharmacother 32:362-375, 1998

2. Vilay AM, Churchwell MD, Mueller BA: Drug metabolism and clearance in acute kidney injury. Crit Care 12:235 (doi: 10.1186/cc7093), 2008

3. Nolin TD, Naud J, Leblond FA, Pichette V: Emerging evidence of the impact of kidney disease on drug metabolism and transport. Clin Pharmacol Ther 83:898-903, 2008

4. Mueller BA, Scarim SK, Macias WL: Comparison of imipenem pharmacokinetics in patients with acute or chronic renal failure treated with continuous hemofiltration. Am J Kidney Dis 21:172-179, 1993

5. Nolin TD, Appiah K, Kendrick SA, Le P, McMonagle E, Himmelfarb J: Hemodialysis acutely improves hepatic CYP3A4 metabolic activity. J Am Soc Nephrol 17:2363-2367, 2006

6. Mueller BA, Pasko DA, Sowinski KM: Higher renal replacement therapy dose delivery influences on drug therapy. Artif Organs 27:808-814, 2003

7. Joy MS, Matzke GR, Frye RF, Palevsky PM: Determinants of vancomycin clearance by continuous venovenous hemofiltration and continuous venovenous hemodialysis. Am J Kidney Dis 31:1019-1027, 1998
8. Churchwell MD, Pasko DA, Mueller BA: Daptomycin clearance during modeled continuous renal replacement therapy. Blood Purif 24:548-554, 2006

9. Uchino S, Cole L, Morimatsu H, Goldsmith D, Bellomo R: Clearance of vancomycin during high-volume haemofiltration: impact of pre-dilution. Intensive Care Med 28:1664-1667, 2002

10. Brunet S, Leblanc M, Geadah D, Parent D, Courteau S, Cardinal J: Diffusive and convective solute clearances during continuous renal replacement therapy at various dialysate and ultrafiltration flow rates. Am J Kidney Dis 34:486-492, 1999

11. Pasko DA, Churchwell MD, Mueller BA: Duration of Continuous Venovenous Hemodialysis and Dialysate Flow Rate on Solute Saturation Coefficient (abstract). European Society of Clinical Pharmacy Conference, 2004, Paris, France

12. Pasko DA, Churchwell MD, Mueller BA: Duration of Continuous Hemofiltration and Ultrafiltration Rate Influence on Sieving Coefficients (abstract). European Society of Clinical Pharmacy Conference, 2004, Paris, France

13. Zaske DE, Cipole RJ, Rotschafer JC, Solem LD, Mosier NR, Strate RG: Gentamicin pharmacokinetics in 1640 patients: methods for control of serum concentrations. Antimicrob Agents Chemother 21: 407-411, 1982

14. Nicolau DP, Freeman CD, Belliveau PP, Nightingale CH, Ross JW, Quintiliani R: Experience with a once-daily aminoglycoside program administered to 2184 adult patients. Antimicrob Agents Chemother 39:650-655, 1995

15. Buijk SE, Mouton JW, Gyssens IC, Verbrugh HA, Bruining HA: Experience with a once-daily dosing program of aminoglycosides in critically ill patients. Intensive Care Med 28:936-942, 2002

16. Dvorchik B, Arbeit RD, Chung J, Liu S, Knebel W, Kastrissios H: Population pharmacokinetics of daptomycin. Antimicrob Agents Chemother 48:2799-2807, 2004

17. Cubist Pharmaceuticals, Inc. Cubicin (Daptomycin for Injection) Package Insert. Lexington MA: Cubist Pharmaceuticals, Inc., 2007.

18. Vilay AM, Grio MC, DePestel DD, Sowinski KM, Gao L, Salama NN, Mueller BA: Daptomycin Pharmacokinetics in Patients Treated With Continuous Venovenous Hemodialysis (CVVHD) (abstract). American Society of Nephrology Renal Week, 2008, Philadelphia, PA

19. Bush LM, Boscia JA, Wendeler M, Pitsakis PG, Kaye D: In vitro postantibiotic effect of daptomycin (LY146032) against Enterococcus faecalis and methicillin-susceptible and methicillin-resistant Staphylococcus aureus strains. Antimicrob Agents Chemother 33:1198-1200, 1989

20. Hanberger H, Nilsson LE, Maller R, Isaksson B: Pharmacodynamics of daptomycin and vancomycin on Enterococcus faecalis and Staphylococcus aureus demonstrated by studies of initial killing and postantibiotic effect and influence of $\mathrm{Ca} 2+$ and albumin on these drugs. Antimicrob Agents Chemother 35:1710-1716, 1991

21. Aronoff GR, Bennett WM, Berns JS, Brier ME, Kasbekar N, Mueller BA, Pasko DA, Smoyer WE: Drug Prescribing in Renal Failure Dosing Guidelines for Adults and Children, 5th edn. Philadelphia: American College of Physicians, 2007

22. Micromedex: Micromedex ${ }^{\circledR}$ Healthcare Series (electronic version). Greenwood Village, CO: Thomson Micromedex. Available at http:/ www.thomsonhc.com, accessed August 26, 2008

23. Vidal L, Shavit M, Fraser A, Paul M, Leibovici L: Systematic comparison of four sources of drug information regarding adjustment of dose for renal function. BMJ 331:263 (doi: 10.1136/ bmj.38476.471088.3A, published May 19, 2005), 2005

24. Aronoff GR: Dose adjustment in renal impairment: response from drug prescribing in renal failure. BMJ 30:331, 2005 